\title{
Double Absorbing Boundaries for Finite-Difference Time-Domain Electromagnetics
}

\author{
John LaGrone ${ }^{\mathrm{a}, 1, *}$, Thomas Hagstrom ${ }^{\mathrm{a}, 1}$ \\ ${ }^{a}$ Department of Mathematics, Southern Methodist University, PO Box 750156, Dallas, \\ TX 75275 .
}

\begin{abstract}
We describe the implementation of optimal local radiation boundary condition sequences for second order finite difference approximations to Maxwell's equations and the scalar wave equation using the double absorbing boundary formulation. Numerical experiments are presented which demonstrate that the design accuracy of the boundary conditions is achieved and, for comparable effort, exceeds that of a convolutional perfectly matched layer with reasonably chosen parameters. An advantage of the proposed approach is that parameters can be chosen using an accurate a priori error bound.
\end{abstract}

Keywords: radiation boundary conditions, Maxwell's equations, wave equation, Yee scheme

\section{Introduction}

An important issue in the simulation of electromagnetic effects is the ability to truncate unbounded domains into regions of interest that can be simulated efficiently and accurately for long times. In the context of finite difference time domain (FDTD) solvers, this typically takes the form of a perfectly matched layer (PML) [1]. The PML method is attractive because it is effective and easy to implement; however, the performance is closely tied to a selection of parameters. While work has been done to automate

\footnotetext{
${ }^{*}$ Corresponding author.

Email addresses: jlagrone@smu.edu (John LaGrone), thagstrom@smu.edu (Thomas Hagstrom)

${ }^{1}$ This work was supported by ARO Grants W911NF-09-1-0344, STTR A11A-015-0067 and NSF Grant DMS-1418871. Any opinions, findings, and conclusions or recommendations expressed in this material are those of the authors and do not necessarily reflect the views of the Army Research Office or the National Science Foundation.
} 
the selection of the optimal PML parameters [2], it is often the case that they must be found by experimentation.

Our goal here is to utilize an alternative to PML, namely the complete radiation boundary conditions (CRBC) [3]. Advantages of the CRBC are:

i. A clear notion of convergence, exponential in increasing order;

ii. A sharp a priori error estimate;

iii. A fast and inexpensive method for selecting optimized parameters.

Earlier implementations of CRBCs relied on the decomposition of the solution in terms of characteristic variables at the artificial boundary [4]. Due to the staggered grid in time and space used in FDTD methods, it is not readily apparent how to directly implement a CRBC type boundary condition. To deal with these staggered grids, we show how a Double Absorbing Boundary (DAB) [5] can be used to leverage the desirable properties of the CRBCs in FDTD simulations. The DAB is constructed by forming a thin non-reflecting layer on which we apply the CRBC on two parallel boundaries. Here we formulate the DAB for the standard second-order central difference approximation to the scalar wave equation satisfied by the components of the electric field, using a 3-point DAB layer. The updated electric field components are then used as boundary data for the staggered grid scheme.

We demonstrate the performance of the method with numerical experiments for problems in waveguides, between parallel plates, and in free space.

\section{Double Absorbing Boundary Method}

\section{1. $C R B C$ Review}

In order to introduce the $\mathrm{DAB}$ method, we first recall that the CRBC [3] is defined recursively using the auxiliary variables $u_{0}, \ldots, u_{P}$

$$
\bar{a}_{j} \frac{\partial u_{j+1}}{\partial t}-\frac{\partial u_{j+1}}{\partial \hat{n}}+\bar{\sigma}_{j} u_{j+1}=a_{j} \frac{\partial u_{j}}{\partial t}+\frac{\partial u_{j}}{\partial \hat{n}}+\sigma_{j} u_{j}
$$

where $u_{0}$ is the solution in the volume and $\hat{n}$ is the outward pointing normal. The parameters $a_{j}, \bar{a}_{j}, \sigma_{j}$, and $\bar{\sigma}_{j}$ are defined as

$$
a_{j}=\frac{\cos \theta_{j}}{c}, \quad \bar{a}_{j}=\frac{\cos \bar{\theta}_{j}}{c}, \quad \sigma_{j}=\frac{1}{c T} \frac{\sin ^{2} \theta_{j}}{\cos \theta_{j}}, \quad \bar{\sigma}_{j}=\frac{1}{c T} \frac{\sin ^{2} \bar{\theta}_{j}}{\cos \bar{\theta}_{j}}
$$


where the angle variables $\theta_{j}$ and $\bar{\theta}_{j}$ are chosen to minimize the reflections, $c$ is the wave speed, and $T$ is the total time of interest (e.g. the simulation time). Here we assume $u_{0}$ satisfies the scalar wave equation in the vicinity of the artificial boundary and beyond:

$$
\frac{\partial^{2} u_{0}}{\partial t^{2}}-c^{2} \nabla^{2} u_{0}=0
$$

After defining a separation parameter $\delta$ to be the minimum separation between the boundary and any scatterers, sources, or other inhomogeneities, in [3] it is shown that the maximum reflection coefficient is given by

$$
\rho=\max _{0 \leq \theta<\pi / 2}\left(\prod_{j=0}^{P-1} \frac{\left|\cos \theta-a_{j}\right|\left|\cos \theta-\bar{a}_{j}\right|}{\left(\cos \theta+a_{j}\right)\left(\cos \theta+\bar{a}_{j}\right)}\right) \cdot\left(\frac{1-\cos \theta}{1+\cos \theta}\right) e^{-\frac{\delta}{c T} \frac{1}{\cos \theta}}
$$

An optimization scheme can be employed to rapidly select the parameters $a_{j}$ and $\bar{a}_{j}$ that minimize $\rho$. It can be proven that $\rho$ decreases exponentially with $P$. Precisely,

$$
P \propto \ln \left(\frac{1}{\rho}\right) \cdot \ln \left(\frac{c T}{\delta}\right)
$$

Functions which compute these parameters are included in our CRBC software library rbcpack (www.rbcpack.org). We emphasize that the angle variables are not incidence angles of plane waves. Rather they parameterize a complete wave representation as derived in [3]. As such the reflection coefficient $\rho$ provides an a priori error estimate. More precisely, since $\rho$ is an upper bound for the reflection error along an entire inversion contour for a Fourier-Laplace representation of the solution in the far field, a direct application of Parseval's relation implies a simple error bound which is attained for some data. Moreover, as the optimization is based on the equidistribution principle, one expects it to be rather accurate for broadband signals. We will see in the numerical experiments that the predicted accuracy can be attained for sufficiently well-resolved waves and that the error bounds are sharp.

\subsection{DAB for the Scalar Wave Equation}

Following from [5], to illustrate the DAB method, we consider the scalar wave equation in the semi-bounded domain as shown in Fig. 1(a)

$$
\frac{\partial^{2} u}{\partial t^{2}}-c^{2} \nabla^{2} u=f
$$


(a)

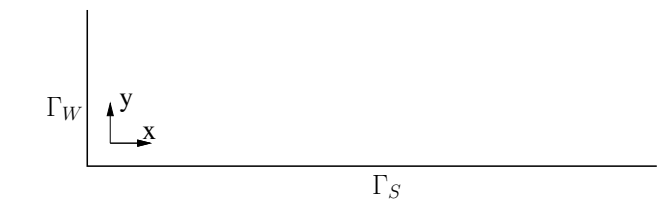

(b)

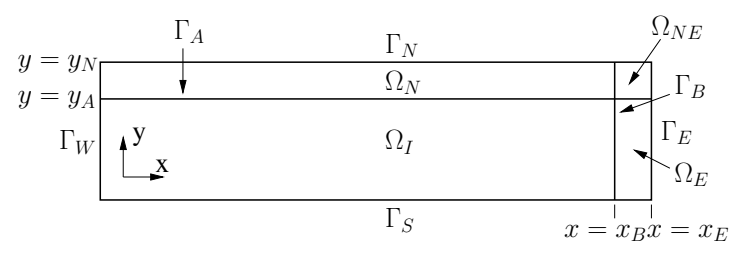

Figure 1: (a) the original domain, (b) the truncated domain

For simplicity, we prescribe zero Dirichlet conditions on the boundaries

$$
u=0, \quad \text { on } \Gamma_{W}, \Gamma_{S},
$$

and initial conditions

$$
\begin{aligned}
u(x, y, t=0) & =h(x, y), \\
\frac{\partial u}{\partial t}(x, y, t=0) & =g(x, y),
\end{aligned}
$$

where $h$ and $g$ are known functions satisfying the boundary conditions. We further require that $c$ is constant, $f=0$, and the initial values vanish outside a compact region, $\Omega_{0}$.

We next truncate the semi-infinite domain by introducing two artificial boundaries: $\Gamma_{N}$ and $\Gamma_{E}$ located at $y=y_{N}$ and $x=x_{E}$, respectively. We additionally introduce two interfaces $\Gamma_{A}$ and $\Gamma_{B}$ which are located at $y=$ $y_{A}<y_{N}$ and $x=x_{B}<x_{E}$, respectively. The entire computation domain, $\Omega$, is bounded by $\Gamma_{N} \bigcup \Gamma_{E} \bigcup \Gamma_{S} \bigcup \Gamma_{W}$. As shown in Fig 1(b), this divides the computational domain into four subdomains: the interior domain $\Omega_{I}$; two thin edge layers, $\Omega_{N}$ and $\Omega_{E}$; and a thin corner layer $\Omega_{N E}$. We suppose the interfaces $\Gamma_{A}$ and $\Gamma_{B}$ are chosen such that $\Omega_{0} \subset \Omega_{I}$; therefore, in the layers $\Omega_{N}, \Omega_{E}$, and $\Omega_{N E}, c$ is constant, $f=0$, and the initial conditions are zero.

The idea is to use the thin layers $\Omega_{N}, \Omega_{E}$, and $\Omega_{N E}$ as absorbing layers so that we may compute the solution in $\Omega_{I}$ as close to the solution of the semi-infinite problem as possible. To do this, we introduce a set of auxiliary variables in each of the layers. In $\Omega_{N}$, we introduce $\phi_{0}, \ldots, \phi_{Q}$; in $\Omega_{E}$ we introduce $v_{0}, \ldots, v_{P}$; and in $\Omega_{N E}$ we introduce the doubly indexed set of 
auxiliary variables $\psi_{p, q}$ with $p=0, \ldots, P$ and $q=0, \ldots, Q$. In each layer, we require the auxiliary variables to satisfy (6) with $f=0$ :

$$
\begin{aligned}
& \frac{\partial^{2} \phi_{q}}{\partial t^{2}}-c^{2} \nabla^{2} \phi_{q}=0, \quad \text { in } \Omega_{N}, \\
& \frac{\partial^{2} v_{p}}{\partial t^{2}}-c^{2} \nabla^{2} v_{p}=0, \quad \text { in } \Omega_{E}, \\
& \frac{\partial^{2} \psi_{p, q}}{\partial t^{2}}-c^{2} \nabla^{2} \psi_{p, q}=0, \quad \text { in } \Omega_{N E} .
\end{aligned}
$$

We insist that the auxiliary variables satisfy zero initial conditions and we apply the same boundary conditions as $u$ on $\Gamma_{S}$ and $\Gamma_{W}$ :

$$
\begin{array}{ll}
\phi_{q}=0, & \text { on } \Gamma_{W}, \\
v_{p}=0, & \text { on } \Gamma_{S} .
\end{array}
$$

In order to couple the auxiliary variables in the layers and the interior, we require that the CRBC recursions (1) be satisfied on the boundaries:

$$
\begin{aligned}
& \left(\bar{a}_{q} \frac{\partial}{\partial t}-\frac{\partial}{\partial y}+\bar{\sigma}_{q}\right) \phi_{q+1}=\left(a_{q} \frac{\partial}{\partial t}+\frac{\partial}{\partial y}+\sigma_{q}\right) \phi_{q}, \text { on } \Gamma_{A}, \Gamma_{N}, \\
& \left(\bar{b}_{p} \frac{\partial}{\partial t}-\frac{\partial}{\partial x}+\bar{\varsigma}_{p}\right) v_{p+1}=\left(b_{p} \frac{\partial}{\partial t}+\frac{\partial}{\partial x}+\varsigma_{p}\right) v_{p}, \text { on } \Gamma_{B}, \Gamma_{E}
\end{aligned}
$$

and for the auxiliary variables defined on the corner layer, $\Gamma_{N E}$, we require

$$
\begin{aligned}
\left(\bar{a}_{q} \frac{\partial}{\partial t}-\frac{\partial}{\partial y}+\bar{\sigma}_{q}\right) \psi_{p, q+1} & =\left(a_{q} \frac{\partial}{\partial t}+\frac{\partial}{\partial y}+\sigma_{q}\right) \psi_{p, q}, \text { on } \Gamma_{A}, \Gamma_{N}, \\
\left(\bar{b}_{p} \frac{\partial}{\partial t}-\frac{\partial}{\partial x}+\bar{\varsigma}_{p}\right) \psi_{p+1, q} & =\left(b_{p} \frac{\partial}{\partial t}+\frac{\partial}{\partial x}+\varsigma_{p}\right) \psi_{p, q}, \text { on } \Gamma_{B}, \Gamma_{E} .
\end{aligned}
$$

To begin the recursions on the interior sides of the layer, we require the slope and value of $\phi_{0}$ and $v_{0}$ to coincide with $u$ :

$$
\begin{array}{llll}
\phi_{0}=u, & \frac{\partial \phi_{0}}{\partial y}=\frac{\partial u}{\partial y}, & & \text { on } \Gamma_{A}, \\
v_{0}=u, & \frac{\partial v_{0}}{\partial x}=\frac{\partial u}{\partial x}, & & \text { on } \Gamma_{B} .
\end{array}
$$

In the corner layer, we require the zeroth level of the auxiliary variable in each direction to agree in slope and value with the auxiliary variables in the 
neighboring layer,

$$
\begin{aligned}
& \psi_{p, 0}=\phi_{p}, \quad \frac{\partial \psi_{p, 0}}{\partial x}=\frac{\partial \phi_{p}}{\partial x}, \quad p=0, \ldots, P, \quad \text { on } \Gamma_{B}, \\
& \psi_{0, q}=v_{q}, \quad \frac{\partial \psi_{0, q}}{\partial y}=\frac{\partial v_{q}}{\partial y}, \quad q=0, \ldots, Q, \quad \text { on } \Gamma_{A} .
\end{aligned}
$$

On the exterior sides of the layers, we terminate the recursions using the Sommerfeld conditions

$$
\begin{aligned}
& \left(\frac{\partial}{\partial t}+c \frac{\partial}{\partial y}\right) \phi_{Q}=0, \quad \text { on } \Gamma_{N}, \\
& \left(\frac{\partial}{\partial t}+c \frac{\partial}{\partial x}\right) v_{P}=0, \quad \text { on } \Gamma_{E}, \\
& \left(\frac{\partial}{\partial t}+c \frac{\partial}{\partial y}\right) \psi_{p, Q}=0, \quad p=0, \ldots, P, \quad \text { on } \Gamma_{N}, \\
& \left(\frac{\partial}{\partial t}+c \frac{\partial}{\partial x}\right) \psi_{P, q}=0, \quad q=0, \ldots, Q, \quad \text { on } \Gamma_{E} .
\end{aligned}
$$

Since the auxiliary variables are continuous across the interfaces at the zeroth level by construction and satisfy the same wave equation as $u$, we have

$$
\begin{aligned}
\phi_{0} & \equiv u, & & \text { in } \Omega_{N}, \\
v_{0} & \equiv u, & & \text { in } \Omega_{E}, \\
\psi_{0,0} & \equiv u, & & \text { in } \Omega_{N E} .
\end{aligned}
$$

We note that the extension to a full three dimensional problem is analogous and simply requires the introduction of a set of triply indexed auxiliary variables to handle corners.

\subsection{Discretization}

To discretize the DAB, we use finite differences in space and time. We define the grid spacings in $x$ and $y$ to be $h_{x}$ and $h_{y}$, respectively, and discretize time with the time-step size $\Delta t$. In order to more efficiently write 
the finite difference scheme, we introduce the following discrete operators:

Forward average in space: $A_{x}^{+} v_{i, j}=\left(v_{i, j}+v_{i+1, j}\right) / 2$,

Forward average in time: $A_{t}^{+} v^{n}=\left(v^{n}+v^{n+1}\right) / 2$,

Forward difference in space: $D_{x}^{+} v_{i, j}=\left(v_{i+1, j}-v_{i, j}\right) / h_{x}$,

Backward difference in space: $D_{x}^{-} v_{i, j}=\left(v_{i, j}-v_{i-1, j}\right) / h_{x}$,

Forward difference in time: $D_{t}^{+} v^{n}=\left(v^{n+1}-v^{n}\right) / \Delta t$,

Backward difference in time: $D_{t}^{-} v^{n}=\left(v^{n}-v^{n-1}\right) / \Delta t$,

and analogously define $A_{y}^{+}, D_{y}^{+}$, and $D_{y}^{-}$. Then we can discretize the interior of the problem (6), $\Gamma_{I}$, using standard second order central differences

$$
\begin{gathered}
D_{t}^{+} D_{t}^{-} u_{i, j}^{n}=c^{2}\left(D_{x}^{+} D_{x}^{-}+D_{y}^{+} D_{y}^{-}\right) u_{i, j}^{n}+f_{i, j}^{n}, \\
i=1, \ldots, n_{x}-1, \quad j=1, \ldots, n_{y}-1, \quad n \geq 1,
\end{gathered}
$$

where there are $\left(n_{x}+1\right)$ and $\left(n_{y}+1\right)$ grid points in the $x$ and $y$ directions, respectively, and $u_{i, j}^{n} \approx u\left(x_{i}, y_{j}, n \Delta t\right), f_{i, j}^{n}=f\left(x_{i}, y_{j}, n \Delta t\right)$, and $\Delta t$ has been chosen to satisfy the CFL condition

$$
\Delta t \leq c^{-1}\left(\left(h_{x}\right)^{-2}+\left(h_{y}\right)^{-2}\right)^{-1 / 2} .
$$

We note that setting $u_{i, j}^{n}=0$ satisfies the boundary conditions whenever $i=0$ or $j=0$, so these values do not require updates.

To discretize the layers $\Omega_{E}, \Omega_{N}$, and $\Omega_{N E}$, we note that they only need to be wide enough to support the wave equation stencil. In the case of second order centered differences, this means that we only require the layers to be three grid points (or $2 h$ ) wide. The layers can be made thicker, but there is no practical benefit 5 . Therefore, $\Omega_{N}$ has $\left(n_{x}+1\right)$ gridpoints in the $x$ direction and 3 gridpoints in the $y$ direction; $\Omega_{E}$ has 3 gridpoints in the $x$ direction and $\left(n_{y}+1\right)$ gridpoints in the $y$ direction; and $\Omega_{N E}$ has 3 gridpoints in each direction. For clarity we index these points in accordance with the interior indexing; for example in $\Omega_{N}$ the auxiliary functions have a second index ranging from $n_{y}-1$ to $n_{y}+1$. Then the updates for the 
interiors of the layers are given by discretizing (10)-(12):

$$
\begin{array}{r}
D_{t}^{+} D_{t}^{-} \phi_{i, n_{y}, q}^{n}=c^{2}\left(D_{x}^{+} D_{x}^{-}+D_{y}^{+} D_{y}^{-}\right) \phi_{i, n_{y}, q}^{n}, \\
i=1, \ldots, n_{x}-1, \quad q=0, \ldots, Q, \\
D_{t}^{+} D_{t}^{-} v_{n_{x}, j, p}^{n}=c^{2}\left(D_{x}^{+} D_{x}^{-}+D_{y}^{+} D_{y}^{-}\right) v_{n_{x}, j, p}^{n}, \\
j=1, \ldots, n_{y}-1, \quad p=0, \ldots, P, \\
D_{t}^{+} D_{t}^{-} \psi_{n_{x}, n_{y}, p, q}^{n}=c^{2}\left(D_{x}^{+} D_{x}^{-}+D_{y}^{+} D_{y}^{-}\right) \psi_{n_{x}, n_{y}, p, q}^{n} \\
q=0, \ldots, Q, \quad p=0, \ldots, P,
\end{array}
$$

The CRBC recursions on the interface side of the layers given in 15$)-(16)$ are discretized as

$$
\begin{array}{r}
\left(\bar{a}_{q} D_{t}^{+} A_{y}^{+}-D_{y}^{+} A_{t}^{+}+\bar{\sigma}_{q} A_{t}^{+} A_{y}^{+}\right) \phi_{i, j, q+1}^{n}= \\
\left(a_{q} D_{t}^{+} A_{y}^{+}+D_{y}^{+} A_{t}^{+}+\sigma_{q} A_{t}^{+} A_{y}^{+}\right) \phi_{i, j, q}^{n}, \\
i=1, \ldots, n_{x}-1, \quad j=n_{y}-1, n_{y}, \\
\left(\bar{b}_{p} D_{t}^{+} A_{x}^{+}-D_{x}^{+} A_{t}^{+}+\bar{\varsigma}_{p} A_{t}^{+} A_{x}^{+}\right) v_{i, j, p+1}^{n}= \\
\left(b_{p} D_{t}^{+} A_{x}^{+}+D_{x}^{+} A_{t}^{+}+\varsigma_{p} A_{t}^{+} A_{x}^{+}\right) v_{i, j, p}^{n}, \\
i=n_{x}-1, n_{x}, \quad j=1, \ldots, n_{y}-1 .
\end{array}
$$

The recursions on the sides of the corner layer $\Omega_{N E}$ given in $(17)-(18)$ are discretized analogously as

$$
\begin{array}{r}
\left(\bar{a}_{q} D_{t}^{+} A_{y}^{+}-D_{y}^{+} A_{t}^{+}+\bar{\sigma}_{q} A_{t}^{+} A_{y}^{+}\right) \psi_{n_{x}, j, p, q+1}^{n}= \\
\left(a_{q} D_{t}^{+} A_{y}^{+}+D_{y}^{+} A_{t}^{+}+\sigma_{q} A_{t}^{+} A_{y}^{+}\right) \psi_{n_{x}, j, p, q}^{n}, \quad j=n_{y}-1, n_{y}, \\
\left(\bar{b}_{p} D_{t}^{+} A_{x}^{+}-D_{x}^{+} A_{t}^{+}+\bar{\varsigma}_{p} A_{t}^{+} A_{x}^{+}\right) \psi_{i, n_{y}, p+1, q}^{n}= \\
\left(b_{p} D_{t}^{+} A_{x}^{+}+D_{x}^{+} A_{t}^{+}+\varsigma_{p} A_{t}^{+} A_{x}^{+}\right) \psi_{i, n_{y}, p, q}^{n}, \quad i=n_{x}-1, n_{x},
\end{array}
$$

We can solve the "forward" recursions on $\Gamma_{A}$ and $\Gamma_{B}$ from $(36)-(39)$ and for $\phi_{i, n_{y}-1, q+1}^{n+1}, v_{n_{x}-1, j, p+1}^{n+1}, \psi_{n_{x}, n_{y}-1, p, q+1}^{n+1}$, and $\psi_{n_{x}-1, n_{y}, p+1, q}^{n+1}$. Similarly, we can solve the "backward" recursions on $\Gamma_{N}$ and $\Gamma_{E}$ for $\phi_{i, n_{y}+1, q}^{n+1}, v_{n_{x}+1, j, p}^{n+1}$, $\psi_{n_{x}, n_{y}+1, p, q}^{n+1}$, and $\psi_{n_{x}+1, n_{y}, p, q}^{n+1}$. We note this yields explicit update formulas, which we enumerate in Appendix A.

Finally, the termination conditions $(23)-(26)$ are discretized as

$$
\begin{array}{rlrl}
\left(D_{t}^{+} A_{y}^{+}+c D_{y}^{+} A_{t}^{+}\right) \phi_{i, n_{y}+1, Q}^{n} & =0, & i=1, \ldots, n_{x}-1, \\
\left(D_{t}^{+} A_{x}^{+}+c D_{x}^{+} A_{t}^{+}\right) v_{n_{x}+1, j, P}^{n} & =0, & j=1, \ldots, n_{y}-1, \\
\left(D_{t}^{+} A_{y}^{+}+c D_{y}^{+} A_{t}^{+}\right) \psi_{n_{x}, n_{y}+1, p, Q}^{n} & =0, & & p=0, \ldots P \\
\left(D_{t}^{+} A_{x}^{+}+c D_{x}^{+} A_{t}^{+}\right) \psi_{n_{x}+1, n_{y}, P, q}^{n} & =0, & & q=0, \ldots Q .
\end{array}
$$


Again, we can solve the equations (40)-(43) to yield explicit updates for $\phi_{i, n_{y}+1, Q}^{n+1}, v_{n_{x}+1, j, P}^{n+1}, \psi_{n_{x}, n_{y}+1, p, Q}^{n+1}$, and $\psi_{n_{x}+1, n_{y}, P, q}^{n+1}$

Then the full update procedure is:

1. Update the internal grid points using (31).

2. Copy the new internal values to zeroth level of the auxiliary variables. Specifically, copy $v_{i, n_{y}-1}^{n+1}$ to $\phi_{i, n_{y}-1,0}^{n+1}$ and copy $u_{n_{x}-1, j}^{n+1}$ to $v_{n_{x}-1, j, 0}^{n+1}$.

3. Update the internal points of $\Omega_{N}$ and $\Omega_{E}$ using (33)-(34).

4. Apply the termination conditions (40)-41) to the points in $\Omega_{N}$ and $\Omega_{E}$.

5. Update the points in $\Omega_{N}$ and $\Omega_{E}$ using the recursions (36)-(37).

6. Copy the updated auxiliary variables from $\Omega_{N}$ and $\Omega_{E}$ to the zeroth level auxiliary variables in $\Omega_{N E}$. Specifically, copy $\phi_{n_{x}-1, n_{y}, q}^{n+1}$ to $\psi_{n_{x}-1, n_{y}, 0, q}^{n+1}$ and $v_{n_{x}, n_{y}-1, p}^{n+1}$ to $\psi_{n_{x}, n_{y}-1, p, 0}^{n+1}$.

7. Compute the updates to $\psi_{n_{x}, n_{y}, p, q}^{n+1}$ using (35).

8. Apply the termination conditions $42-(43)$ to the points in $\Omega_{N E}$.

9. Apply the recursive updates $(38)-(39)$.

10. Copy the updated values from $\Omega_{N E}$ into $\Omega_{N}$ and $\Omega_{E}$. In particular, copy $\psi_{n_{x}, n_{y}, 0, q}^{n+1}$ to $\phi_{n_{x}, n_{y}, q}^{n+1}$ and $\psi_{n_{x}, n_{y}, p, 0}^{n+1}$ to $v_{n_{x}, n_{y}, p}^{n+1}$.

11. Copy the updated values from $\Omega_{N}$ and $\Omega_{E}$ into $\Omega_{I}$. In particular, copy $\phi_{i, n_{y}, 0}^{n+1}$ to $u_{i, n_{y}}^{n+1}$ and $v_{n_{x}, j, 0}^{n+1}$ to $u_{n_{x}, j}^{n+1}$.

12. Move to the next time step $n \leftarrow n+1$.

Notice that we only require updates to the points in the wave equation's stencil. In particular, we do not need to update the points in the corners of $\Omega_{N E}$. Finally, note that we can handle other boundary condition types by modifying the wave equation updates (33)-34. For example, if on $\Gamma_{W}$ we instead imposed a zero Neumann condition, we would modify the wave equation updates at $\Gamma_{W}$ in $\Omega_{N}$ to be

$$
D_{t}^{+} D_{t}^{-} \phi_{0, n_{y}, q}^{n}=c^{2}\left(\frac{\phi_{1, n_{y}, q}^{n}-\phi_{0, n_{y}, q}^{n}}{h^{2}}+D_{y}^{+} D_{y}^{-} \phi_{0, n_{y}, q}^{n}\right), \quad q=0, \ldots, Q,
$$

which we arrive at by substituting $D_{x}^{+} \phi_{0, n_{y}, q}^{n}=0$.

\section{Application to the Yee Scheme}

In order to apply the DAB to the Yee scheme, we require the material in the neighborhood of the boundaries to be homogeneous, isotropic, and 
dielectric. In this case, Maxwell's equations are given by

$$
\begin{aligned}
\frac{\partial \mathbf{H}}{\partial t} & =-\frac{1}{\mu} \nabla \times \mathbf{E}, \\
\frac{\partial \mathbf{E}}{\partial t} & =\frac{1}{\varepsilon} \nabla \times \mathbf{H},
\end{aligned}
$$

subject to the constraints

$$
\begin{aligned}
\nabla \cdot \mathbf{E} & =0 \\
\nabla \cdot \mathbf{H} & =0
\end{aligned}
$$

We emphasize that there can be any number of sources, scatterers, or other inhomogeneities in the interior of the domain so long as they are separated from the DAB boundaries by some distance $\delta>0$.

To discretize these equations using Yee's scheme [6] on a rectangular domain $\left[x_{L}, x_{R}\right] \times\left[y_{L}, y_{R}\right] \times\left[z_{L}, z_{R}\right]$ with mesh spacings of $h_{x}, h_{y}$, and $h_{z}$, in the $x, y$, and $z$ directions, respectively, we define

$$
x_{i}=x_{L}+i h_{x}, \quad y_{j}=y_{L}+j h_{y}, \quad z_{k}=z_{L}+k h_{z} .
$$

We choose a time step size, $\Delta t$, satisfying

$$
\Delta t \leq c^{-1}\left(\left(h_{x}\right)^{-2}+\left(h_{y}\right)^{-2}+\left(h_{z}\right)^{-2}\right)^{-1 / 2},
$$

with the wave speed, $c=(\varepsilon \mu)^{-1 / 2}$. Letting

$$
t_{n}=n \Delta t
$$

Maxwell's equations are approximated on a staggered space-time grid:

$$
\begin{gathered}
D_{t}^{+}\left(H_{x}\right)_{i, j+1 / 2, k+1 / 2}^{n}=\frac{1}{\mu}\left(D_{z}^{+}\left(E_{y}\right)_{i, j+1 / 2, k}^{n+1 / 2}-D_{y}^{+}\left(E_{z}\right)_{i, j, k+1 / 2}^{n+1 / 2}\right), \\
D_{t}^{+}\left(H_{y}\right)_{i+1 / 2, j, k+1 / 2}^{n}=\frac{1}{\mu}\left(D_{x}^{+}\left(E_{z}\right)_{i, j, k+1 / 2}^{n+1 / 2}-D_{z}^{+}\left(E_{x}\right)_{i+1 / 2, j, k}^{n+1 / 2}\right), \\
D_{t}^{+}\left(H_{z}\right)_{i+1 / 2, j+1 / 2, k}^{n}=\frac{1}{\mu}\left(D_{y}^{+}\left(E_{x}\right)_{i+1 / 2, j, k}^{n+1 / 2}-D_{x}^{+}\left(E_{y}\right)_{i, j+1 / 2, k}^{n+1 / 2}\right), \\
D_{t}^{-}\left(E_{x}\right)_{i+1 / 2, j, k}^{n+1 / 2}=\frac{1}{\varepsilon}\left(D_{y}^{-}\left(H_{z}\right)_{i+1 / 2, j+1 / 2, k}^{n}-D_{z}^{-}\left(H_{y}\right)_{i+1 / 2, j, k+1 / 2}^{n}\right), \\
D_{t}^{-}\left(E_{y}\right)_{i, j+1 / 2, k}^{n+1 / 2}=\frac{1}{\varepsilon}\left(D_{z}^{-}\left(H_{x}\right)_{i, j+1 / 2, k+1 / 2}^{n}-D_{x}^{-}\left(H_{z}\right)_{i+1 / 2, j+1 / 2, k}^{n}\right), \\
D_{t}^{-}\left(E_{z}\right)_{i, j, k+1 / 2}^{n+1 / 2}=\frac{1}{\varepsilon}\left(D_{x}^{-}\left(H_{y}\right)_{i+1 / 2, j, k+1 / 2}^{n}-D_{y}^{-}\left(H_{x}\right)_{i, j+1 / 2, k+1 / 2}^{n}\right) .
\end{gathered}
$$


Note that if the initial data is discretely divergence free, it is easy to verify that discretizations of (47) and (48) are automatically enforced by the update scheme. Furthermore, it can be shown that each of the field components satisfies the standard second order central difference approximation to the scalar wave equation, e.g.

$$
D_{t}^{+} D_{t}^{-}\left(E_{x}\right)_{i+1 / 2, j, k}^{n+1 / 2}=c^{2}\left(D_{x}^{+} D_{x}^{-}+D_{y}^{+} D_{y}^{-}+D_{z}^{+} D_{z}^{-}\right)\left(E_{x}\right)_{i+1 / 2, j, k}^{n+1 / 2} .
$$

Therefore, we can use the DAB formulation given in Sec. 2.2 for each $\mathbf{E}$ field component. We want to emphasize that CRBC-type boundary conditions can be directly applied to Maxwell's equations in other discretizations, but it is not readily apparent that this is possible to do in the Yee scheme because of the staggered space-time grid. For instance, CRBCs are successfully applied to a discontinuous Galerkin method in [7] by applying the CRBC recursions (1) to the characteristic equations for Maxwell's equations.

In principle, we should only be required to update the tangential $\mathbf{E}$ field values on the $\mathrm{DAB}$ boundaries to provide the correct number of boundary conditions. However, in the cases where there are DAB layers meeting at edges or corners, we require information from the normal $\mathbf{E}$ field auxiliary components. It is our belief that it should be possible to obtain this information without applying the DAB conditions to the normal $\mathbf{E}$ field component on the boundary (e.g. using the divergence free condition (47)), but our current implementation involves the evolution of all three components on adjacent DAB layers. Full details are given in Appendix B.

\section{Numerical Results}

\subsection{Free Space Transverse Magnetic}

We simulated the free space transverse magnetic problem given by

$$
\begin{array}{r}
\mu \frac{\partial H_{x}}{\partial t}=-\frac{\partial E_{z}}{\partial y} \\
\mu \frac{\partial H_{y}}{\partial t}=\frac{\partial E_{z}}{\partial x} \\
\varepsilon \frac{\partial E_{z}}{\partial t}=\frac{\partial H_{y}}{\partial x}-\frac{\partial H_{x}}{\partial y}
\end{array}
$$

To start the simulation, we take initial data from a solution of the form

$$
E_{z}=\mu \frac{\partial w}{\partial t}, \quad H_{x}=-\frac{\partial w}{\partial y}, \quad H_{y}=\frac{\partial w}{\partial x}
$$


where $w$ is a solution of the scalar wave equation produced by a point source centered at $(0,0.1)$ with the time amplitude $\exp \left(-125(t+.475)^{2}\right)$. The DAB boundaries were placed at $x= \pm 1$ and $y= \pm 1$. The errors reported are relative to the initial condition and are given by the following formula

$$
e_{\text {rel }}^{n}=\sqrt{\frac{\varepsilon\left\|\mathbf{E}_{\text {approx }}^{n+1 / 2}-\mathbf{E}_{\text {exact }}^{n+1 / 2}\right\|_{2}^{2}+\mu\left\|\mathbf{H}_{\text {approx }}^{n}-\mathbf{H}_{\text {exact }}^{n}\right\|_{2}^{2}}{\varepsilon\left\|\mathbf{E}_{\text {exact }}^{1 / 2}\right\|_{2}^{2}+\mu\left\|\mathbf{H}_{\text {exact }}^{0}\right\|_{2}^{2}}}
$$

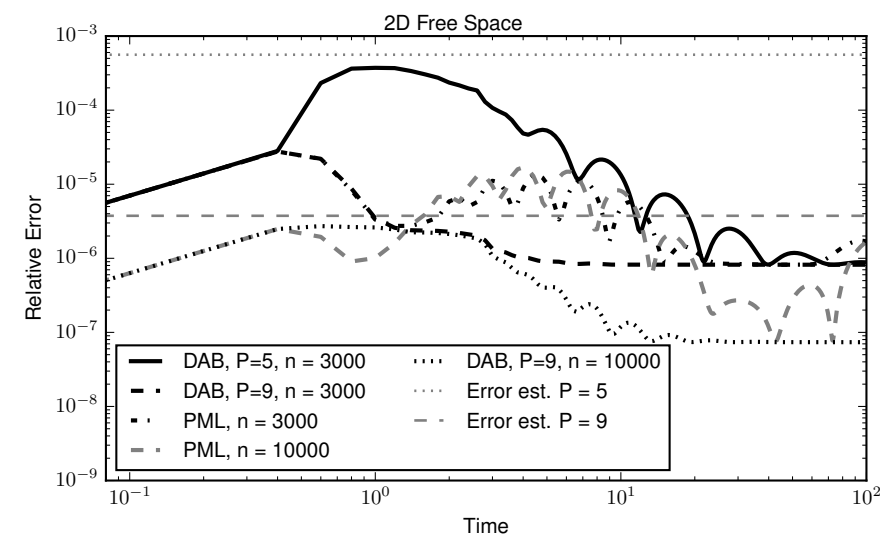

Figure 2: Relative Error in Free Space for the Transverse Magnetic Test Problem.

Table 1: Simulation results for the Transverse Magnetic problem in Free Space.

\begin{tabular}{|c|c|c|c|}
\hline Grid & DoFs & BC & Max Rel Err \\
\hline \hline $3000 \times 3000$ & $2.72 \times 10^{7}$ & DAB P $=5$ & $3.73 \times 10^{-4}$ \\
\hline $3000 \times 3000$ & $2.73 \times 10^{7}$ & DAB P $=9$ & $2.76 \times 10^{-5}$ \\
\hline $10000 \times 10000$ & $3.01 \times 10^{8}$ & DAB P $=9$ & $2.72 \times 10^{-6}$ \\
\hline $3000 \times 3000$ & $2.76 \times 10^{7}$ & PML & $2.76 \times 10^{-5}$ \\
\hline $10000 \times 10000$ & $3.02 \times 10^{8}$ & PML & $1.69 \times 10^{-5}$ \\
\hline
\end{tabular}

Here we compare our results to those obtained using 10-point convolution PML (PML) [8] with parameters $\alpha_{\max }=0.25, \kappa_{\max }=0.15$, and $\sigma_{\max } \approx 5000$ for the coarse grid and $\sigma_{\max } \approx 16700$ for the refined grid. These parameters were selected based on the recommendations from [8]. The proposed formulation with $P=9$ uses fewer degrees-of-freedom than the PML but achieves the same short-time accuracy and better long-time 
accuracy with the same interior grid of $3000^{2} E_{z}$ grid points; see Figure 2 . With further refinement the error is reduced by an order of magnitude and achieves the a priori error estimate. Before the wave reaches the boundary, we see only discretization error. After impact, the boundary error dominates the simulation. This is particularly evident when comparing the simulations with $P=5$ and $P=9$ on the same grid as the difference in error is entirely due to the boundary condition order. We believe that the error in the PML boundary increases after a short time because the wave impacts the exterior boundary of the PML and reflects back into the interior domain, but we note that the overall performance is similar to $P=9$ and may be improved with better PML parameter selection. Finally, the fact that the error decreases in stages for the boundaries is an indication that a wave is reflected and then at least partially absorbed once it travels to the other side of the domain.

\subsection{Transverse Magnetic in a Waveguide}

To test these boundary conditions in a waveguide, we simulated the transverse magnetic problem using the solution

$$
E_{z}=\mu \frac{\partial \phi}{\partial t}, \quad H_{x}=-\frac{\partial \phi}{\partial y}, \quad H_{y}=\frac{\partial \phi}{\partial x},
$$

where $\phi$ is a solution of the scalar wave equation satisfying zero Dirichlet (PEC) boundary conditions at $y=0$ and $y=1$ produced by a point source centered at $(0,0.1)$ with a time amplitude $\exp \left(-125(t+.475)^{2}\right)$. The DAB and PML boundaries were placed at $x= \pm 1$.

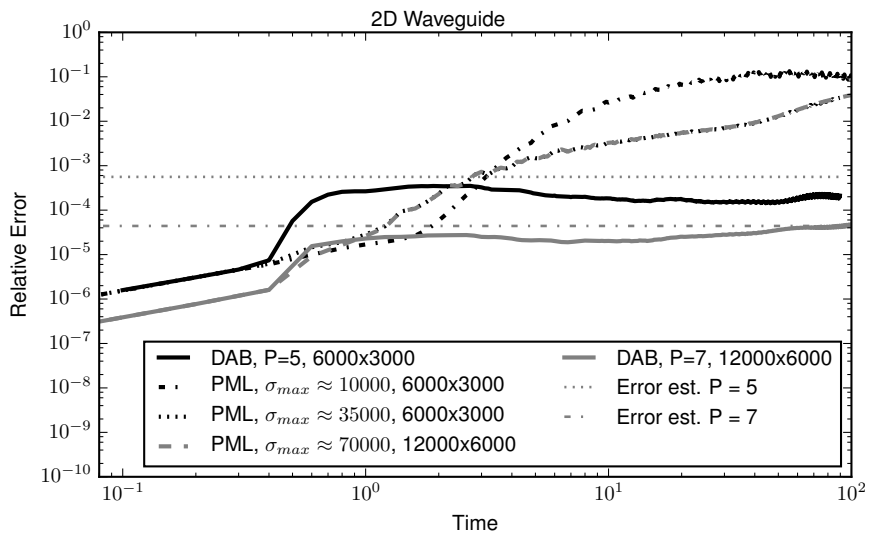

Figure 3: Relative Error for the Transverse Magnetic Test Problem in a Waveguide computed according to 61). Maximum relative errors can be seen in Table 2 
Table 2: Simulation results for the Transverse Magnetic problem in a Waveguide.

\begin{tabular}{|c|c|c|c|}
\hline Grid & DoFs & BC & Max Rel Err \\
\hline \hline $6000 \times 3000$ & $5.41 \times 10^{7}$ & DAB P $=5$ & $3.52 \times 10^{-4}$ \\
\hline $12000 \times 6000$ & $2.16 \times 10^{8}$ & DAB P $=7$ & $4.82 \times 10^{-5}$ \\
\hline $6000 \times 3000$ & $5.43 \times 10^{7}$ & PML, $\sigma_{\max } \approx 35000$ & $3.37 \times 10^{-2}$ \\
\hline $6000 \times 3000$ & $5.43 \times 10^{7}$ & PML, $\sigma_{\max } \approx 10000$ & $1.33 \times 10^{-1}$ \\
\hline $12000 \times 6000$ & $2.17 \times 10^{8}$ & PML, $\sigma_{\max } \approx 70000$ & $3.88 \times 10^{-2}$ \\
\hline
\end{tabular}

Again, we compare our results to a PML with parameters $\alpha_{\max }=0.25$ and $\kappa_{\max }=0.15$ in Figure 3 . We note that we have chosen to do our tests using a convolution PML [8] because it more effective at absorbing evanescent waves than standard PMLs. We show the results for choosing $\sigma_{\max } \approx 35000$, which corresponds to values recommended in [8] and $\sigma_{\max } \approx 10000$ to illustrate the importance of parameter selection for PML accuracy. The refined grid uses $\sigma_{\max } \approx 70000$. Here, we see that the DAB boundaries, which have automated parameter tuning, perform much better than the PML with the parameters tested. We believe that the difference in error between the PML and DAB is due to how well the boundaries handle evanescent waves; error estimates for the Bérenger PML on the continuous level, which can be deduced from the reflection analyses in [9, 10, do indicate that to maintain accuracy over long times in waveguide geometry one must use a thick layer. Enhancements of the Bérenger PML to deal with evanescent modes have been proposed [11, 12, 13], but we do not know how their performance compares with the CPML used in our experiments.

\subsection{D Wave Guide}

For our tests in 3D, we use a solution to Maxwell's equations of the form

$$
\begin{aligned}
\mathbf{E} & =-\mu\left(\nabla \times \frac{\partial \mathbf{W}}{\partial t}\right), \\
\mathbf{H} & =\nabla \times(\nabla \times \mathbf{W}) .
\end{aligned}
$$

We choose $\mathbf{W}$ to be the point source solution to the vector wave equation $\mathbf{W} \equiv \frac{e^{-\gamma(t+\tau-r)^{2}}}{r}[1,1,1]^{T}$, where $\gamma$ and $\tau$ are parameters and $r=\left\|\mathbf{x}-\mathbf{x}_{\text {src }}\right\|$. Using a solution generated from a point source located at $\mathbf{x}_{s r c}=[0.8,0.8,0.8]$ with $\tau=0.39$ and $\gamma=130$, we tested our implementation on the domain $[0,1.6]^{3}$ with DAB boundaries at $x=0$ and $x=1.6$ and PEC boundaries elsewhere. The simulations were run with the DAB parameter $P=3$ and $P=5$ on a grid with $n$ Yee cells in each spatial direction. 


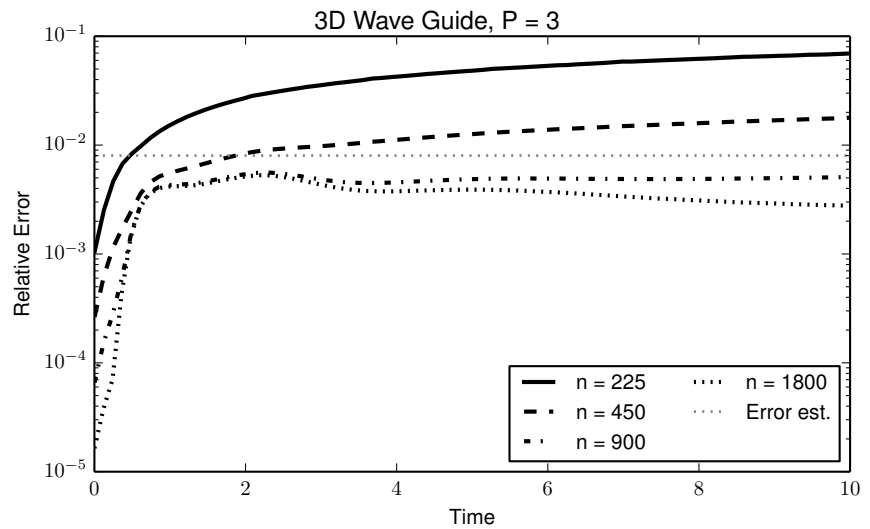

Figure 4: Relative Error for the 3D wave guide problem using DAB boundaries with 3 CRBC recursions.

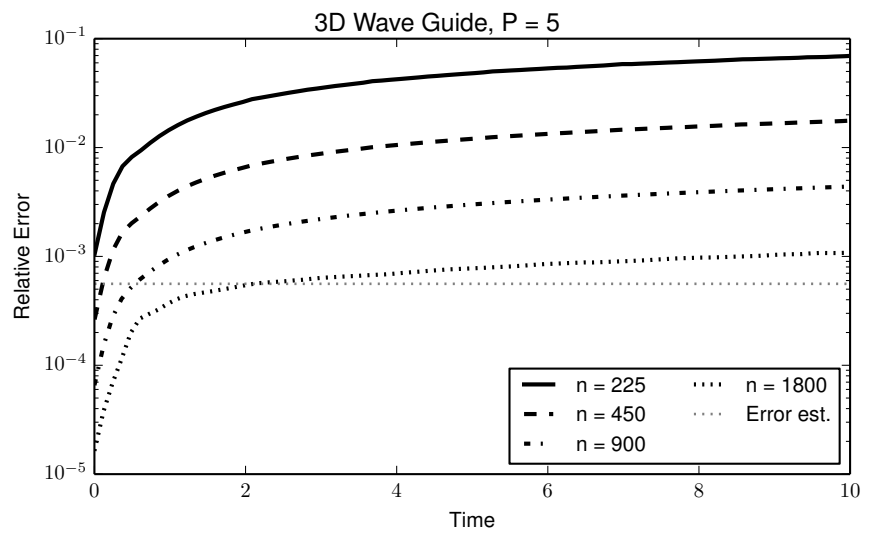

Figure 5: Relative Error for the 3D wave guide problem using DAB boundaries with 5 CRBC recursions.

The results for various mesh spacings are displayed in Figures 4 and 5. We also show the a priori error estimate computed by the optimization routine. We are able to reduce the error below the estimate for $P=3$, which is approximately $1 \%$, using a grid of $900^{3}$. With further refinement using $P=3$, the boundary error dominates and we virtually no improvement in error. For $P=5$ the grid would have to be refined again by a factor of approximately two in order to reduce the error below the estimate. Again we note that the a priori error estimates are achieved on sufficiently fine grids. 


\subsection{Parallel Plates}

Using the same source parameters as in Sec. 4.3, we set DAB boundaries at $x=0, x=1.6, y=0$, and $y=1.6$ and PEC boundaries elsewhere.

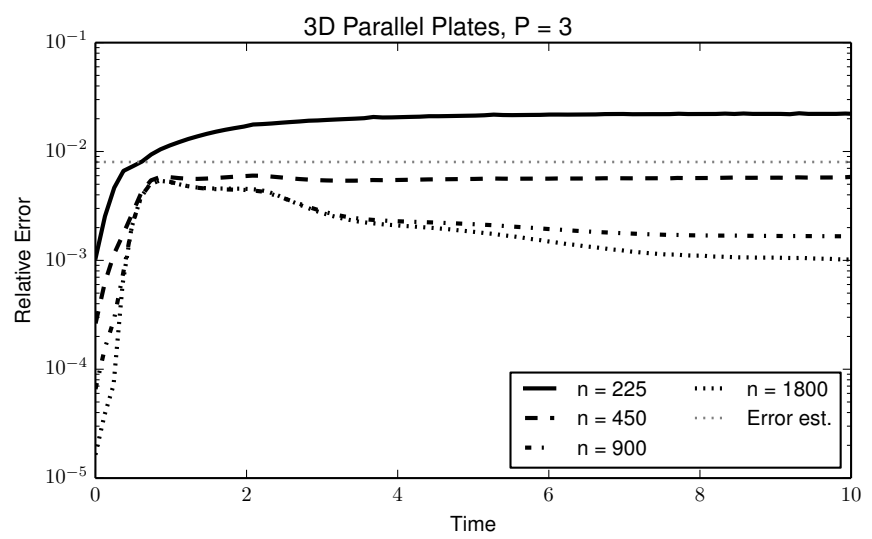

Figure 6: Relative Error for the 3D parallel plate problem using DAB boundaries with 3 CRBC recursions.

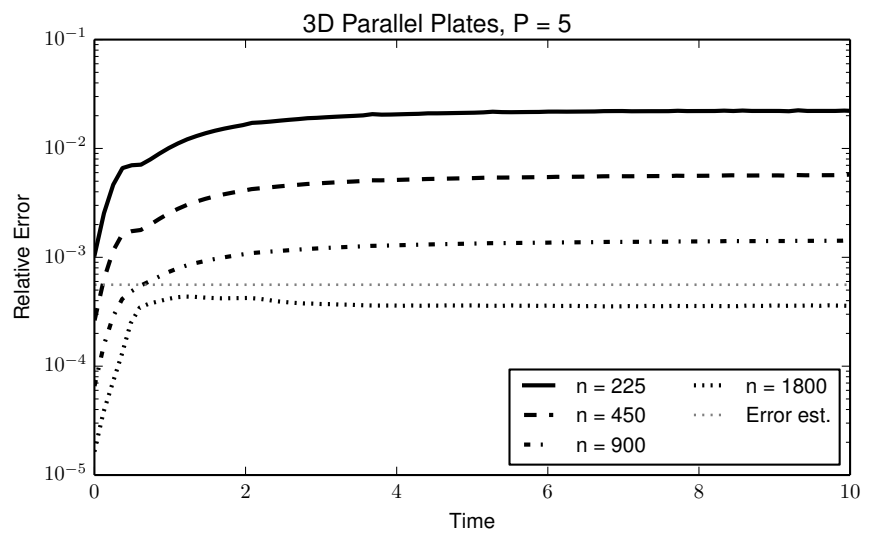

Figure 7: Relative Error for the 3D parallel plate problem using DAB boundaries with 5 CRBC recursions.

In Figures 6 and 7 we see the behavior is similar to the wave guide problem in Figures 4 and 5 but we observe slightly better errors in this case. We believe this is due to the fact that, on average, waves have a shorter residence time in the computational domain and thus experience 
less numerical dispersion. In this case, we are able to reduce the error below the estimate for $P=5$.

\subsection{Free Space}

Again, for the source parameters described Sec. 4.3, we use DAB boundaries on all of the boundary faces.

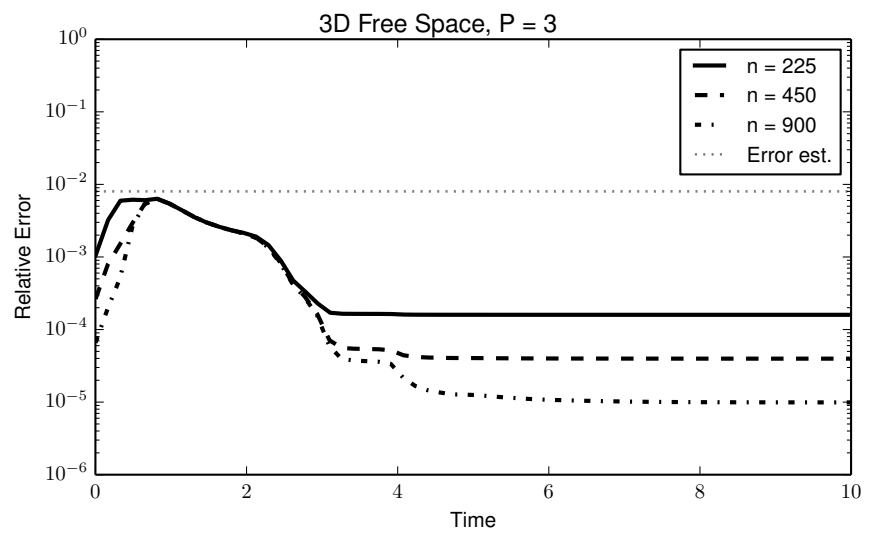

Figure 8: Relative Error for the 3D free space problem using DAB boundaries with 3 CRBC recursions.

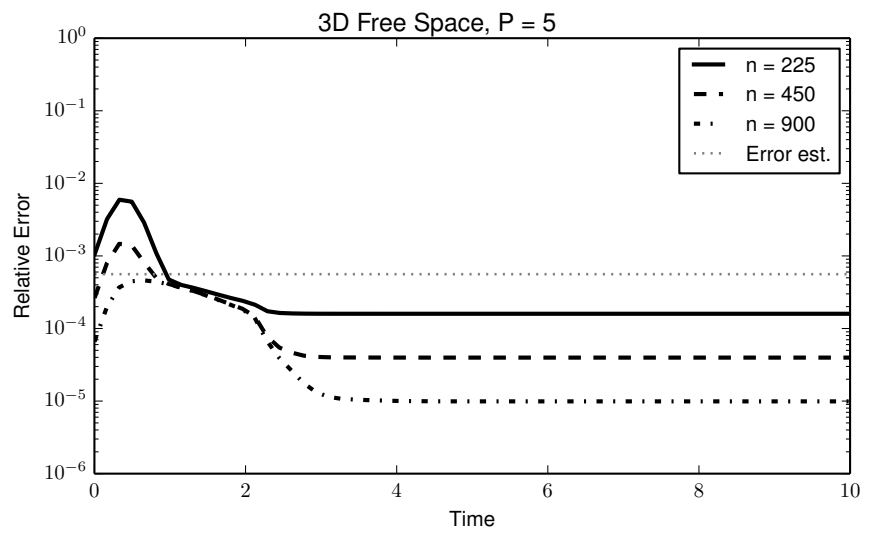

Figure 9: Relative Error for the 3D free space problem using DAB boundaries with 5 CRBC recursions.

In Figures 8 and 9 we see the performance appears to be much better. In terms of error, this is the easiest test case because the waves leave the 
computational domain quickly and impact the boundaries at near-normal incidence. It is worth noting that the stair stepping behavior of the error in Figures 8 and 9 is caused by errors reflecting across the domain and each decrease of error corresponds to the predicted reflection coefficient of the boundary. The errors at long times are the same for both $P=3$ and $P=5$ because the erroneously reflected waves have had ample time to travel across the domain and be absorbed by the boundaries. We then see only the discretization error.

\subsection{Comments on Cost}

Comparing the cost of the DAB layer to PML is not straightforward. Ideally, we would like to compare the accuracy of the DAB and PML boundaries of comparable costs. This is problematic because the performance of the PML is heavily dependent on the selection of parameters, which we again emphasize is an open problem (see e.g. [2]). Nonetheless, we can count the flops assuming that we have precomputed coefficients when possible.

In three dimensions, the PML implementation we have used 8 , has the following costs per node in the tangential directions:

$$
C_{P M L}^{ \pm}(w)=36 w, \quad C_{P M L}^{\times}(w)=24 w,
$$

where $C_{P M L}^{ \pm}(w)$ is the number of addition/subtraction operations and $C_{P M L}^{\times}(w)$ is the number of multiplications as a function of the thickness, $w$; that is the number of points in the normal direction in the layer. We note that other forms of the PML may have significantly different costs and there are potentially large setup costs associated with the selection of good PML parameters.

Similarly, the worst case cost of updating the DAB layer for all three $\mathbf{E}$ field components as a function of the number of recursions, $P$, is given by

$$
C_{D A B}^{ \pm}(P)=60 P+39, \quad C_{D A B}^{\times}(P)=57 P+21 .
$$

We can see that the theoretical computational cost of a PML 10 cells thick is somewhat cheaper than using 5 recursions in the DAB in the worst case scenario. If there are no edges or corners present, then the DAB only needs to update the tangential $\mathbf{E}$ field components, which results in the reduced computational costs

$$
C_{D A B}^{ \pm}(P)=40 P+26, \quad C_{D A B}^{\times}(P)=38 P+14 .
$$

In this case, the DAB with 5 recursions is theoretically cheaper than the PML with a width of 10 . 
We again emphasize that these statements are only meaningful if there is also a guarantee of similar effectiveness for both boundary types. In this regard, we believe the DAB boundaries may often be more efficient in practice because the DAB requires essentially no setup costs associated with finding good parameters. In contrast it may be necessary to run multiple simulations to identify reasonable PML parameters. Moreover in waveguide geometries we have been able to achieve significantly better accuracy than any PML we have tried.

\section{Code}

Code that is capable of reproducing most of the results presented here along with a library to provide the discretization of the DAB boundaries is available for download at http://www.rbcpack.org.

\section{Conclusions and Generalizations}

We have demonstrated a convenient and automatic DAB implementation of CRBCs for electromagnetic waves in homogeneous, dielectric media, using modules from our open-source library. In our experiments for free space problems we have achieved accuracy/cost performance somewhat better than implementations of CPML suggested in the literature, while our results in waveguide geometries were significantly more accurate.

Generalizations of our algorithms to more complex models in the far field are theoretically possible, though we have not yet implemented them for the Maxwell system. In [14] we demonstrate the effectiveness of CRBCs in stratified media using parameters optimized for the maximum wave speed. We expect this approach will work well for layered dielectric media. Anisotropic media typically pose a greater challenge in terms of finding parameters which guarantee exponential convergence and we have not yet considered this problem for electromagnetic waves. This is clearly an important topic for future work.

\section{References}

[1] J.-P. Berenger, A perfectly matched layer for the absorption of electromagnetic waves, Journal of Computational Physics 114 (2) (1994) 185

- 200. doi:http://dx.doi.org/10.1006/jcph.1994.1159.

URL http://www.sciencedirect.com/science/article/pii/ S0021999184711594 
[2] A. Modave, E. Delhez, C. Geuzaine, Optimizing perfectly matched layers in discrete contexts, International Journal for Numerical Methods in Engineering 99 (6) (2014) 410-437. doi:10.1002/nme.4690. URL http://dx.doi.org/10.1002/nme.4690

[3] T. Hagstrom, T. Warburton, Complete radiation boundary conditions: Minimizing the long time error growth of local methods, SIAM Journal on Numerical Analysis 47 (5) (2009) 3678-3704. arXiv: http://epubs. siam.org/doi/pdf/10.1137/090745477, doi:10.1137/090745477. URL http://epubs.siam.org/doi/abs/10.1137/090745477

[4] K. Stein, Complete radiation boundary conditions: Corner and edge closure conditions, Ph.D. thesis, Southern Methodist University (2012).

[5] T. Hagstrom, D. Givoli, D. Rabinovich, J. Bielak, The double absorbing boundary method, Journal of Computational Physics 259 (0) (2014) 220 - 241. doi:http://dx.doi.org/10.1016/j.jcp.2013.11.025.

URL http://www.sciencedirect.com/science/article/pii/ S0021999113007870

[6] K. Yee, Numerical solution of initial boundary value problems involving Maxwell's equations in isotropic media, Antennas and Propagation, IEEE Transactions on 14 (3) (1966) 302-307. doi:10.1109/TAP.1966. 1138693 .

[7] X. R. Chen, D. Appelö, T. Hagstrom, A hybrid Hermite-discontinuous Galerkin method for hyperbolic systems with application to Maxwell's equations, Journal of Computational Physics 257, Part A (2014) 501 520. doi:http://dx.doi.org/10.1016/j.jcp.2013.09.046.

URL http://www.sciencedirect.com/science/article/pii/ S0021999113006608

[8] J. A. Roden, S. D. Gedney, Convolution pml (cpml): An efficient fdtd implementation of the cfspml for arbitrary media, Microwave and Optical Technology Letters 27 (5) (2000) 334-339. doi: 10.1002/1098-2760(20001205) 27:5<334: :AID-MOP14>3.0.CO;2-A. URL http://dx.doi.org/10.1002/1098-2760(20001205)27: 5<334: :AID-MOP14>3.0.CO;2-A

[9] J. Diaz, P. Joly, A time domain analysis of \{PML\} models in acoustics, Computer Methods in Applied Mechanics and Engineering 195 (2932) (2006) 3820 - 3853, absorbing Boundary Conditions. 
doi:http://dx.doi.org/10.1016/j.cma.2005.02.031.

URL http://www.sciencedirect.com/science/article/pii/ S0045782505002604

[10] A. T. de Hoop, P. M. van den Berg, R. F. Remis, Absorbing boundary conditions and perfectly matched layers - an analytic time-domain performance analysis, IEEE Transactions on Magnetics 38 (2) (2002) 657-660. doi:10.1109/20.996171.

[11] Y. C. Lau, M. S. Leong, P. S. Kooi, Extension of berenger's pml boundary condition in matching lossy medium and evanescent waves, Electronics Letters 32 (11) (1996) 974-976. doi:10.1049/el:19960627.

[12] J. Fang, Z. Wu, Generalized perfectly matched layer for the absorption of propagating and evanescent waves in lossless and lossy media, IEEE Transactions on Microwave Theory and Techniques 44 (12) (1996) 2216-2222. doi:10.1109/22.556449.

[13] J. P. Berenger, An effective pml for the absorption of evanescent waves in waveguides, IEEE Microwave and Guided Wave Letters 8 (5) (1998) 188-190. doi:10.1109/75.668706.

[14] T. Hagstrom, High-order radiation boundary conditions for stratified media and curvilinear coordinates, Journal of Computational Acoustics 20 (02) (2012) 1240002. arXiv:http://www. worldscientific.com/doi/pdf/10.1142/S0218396X12400024, doi:10.1142/S0218396X12400024. URL http://www.worldscientific.com/doi/abs/10.1142/ S0218396X12400024

\section{Appendix A. Explicit DAB Update Formulas}

The explicit update formulas for the DAB equations given in Sec. 2.3 are provided here for convenience. The wave equation updates (33)-(35) are of the form

$$
\begin{array}{r}
v_{i, j, p}^{n+1}=2 v_{i, j, p}^{n}-v_{i, j, p}^{n-1}+c(\Delta t)^{2}\left(\frac{v_{i-1, j, p}^{n}-2 v_{i, j, p}^{n}+v_{i+1, j, p}^{n}}{h_{x}^{2}}\right. \\
\left.+\frac{v_{i, j-1, p}^{n}-2 v_{i, j, p}^{n}+v_{i, j+1, p}^{n}}{h_{y}^{2}}\right) .
\end{array}
$$


The recursions (37) and (18) can be solved for an explicit update in the "forward" direction on the interface side, for example recursions in the $\mathrm{x}$-direction:

$$
\begin{array}{r}
\left(\bar{a}_{p}+\frac{\Delta t}{h_{x}}+\frac{\Delta t}{2} \bar{\sigma}_{p}\right) v_{i, j, p+1}^{n+1}=\left(\bar{a}_{p}-\frac{\Delta t}{h_{x}}-\frac{\Delta t}{2} \bar{\sigma}_{p}\right) v_{i, j, p+1}^{n} \\
+\left(\bar{a}_{p}+\frac{\Delta t}{h_{x}}-\frac{\Delta t}{2} \bar{\sigma}_{p}\right) v_{i+1, j, p+1}^{n}+\left(-a_{p}+\frac{\Delta t}{h_{x}}+\frac{\Delta t}{2} \sigma_{p}\right) v_{i+1, j, p}^{n} \\
+\left(-a_{p}-\frac{\Delta t}{h_{x}}+\frac{\Delta t}{2} \sigma_{p}\right) v_{i, j, p}^{n}+\left(-\bar{a}_{p}+\frac{\Delta t}{h_{x}}-\frac{\Delta t}{2} \bar{\sigma}_{p}\right) v_{i+1, j, p+1}^{n+1} \\
+\left(a_{p}+\frac{\Delta t}{h_{x}}+\frac{\Delta t}{2} \sigma_{p}\right) v_{i+1, j, p}^{n+1}+\left(a_{p}-\frac{\Delta t}{h_{x}}+\frac{\Delta t}{2} \sigma_{p}\right) v_{i, j, p}^{n+1} .
\end{array}
$$

The recursions in the y-direction, (36) and (17), are essentially identical, but the differencing is done on the index $j$ instead of $i$.

Similary, the recursions (37) can be solved for an explicit update in the "backward" direction on the interface side, for example recursions in the $\mathrm{x}$-direction:

$$
\begin{array}{r}
\left(a_{p}+\frac{\Delta t}{h_{x}}+\frac{\Delta t}{2} \sigma_{p}\right) v_{i, j, p}^{n+1}=\left(a_{p}-\frac{\Delta t}{h_{x}}-\frac{\Delta t}{2} \sigma_{p}\right) v_{i, j, p}^{n} \\
+\left(a_{p}+\frac{\Delta t}{h_{x}}-\frac{\Delta t}{2} \sigma_{p}\right) v_{i-1, j, p}^{n}+\left(-\bar{a}_{p}+\frac{\Delta t}{h_{x}}+\frac{\Delta t}{2} \bar{\sigma}_{p}\right) v_{i-1, j, p+1}^{n} \\
+\left(-\bar{a}_{p}-\frac{\Delta t}{h_{x}}+\frac{\Delta t}{2} \bar{\sigma}_{p}\right) v_{i, j, p+1}^{n}+\left(-a_{p}+\frac{\Delta t}{h_{x}}-\frac{\Delta t}{2} \sigma_{p}\right) v_{i-1, j, p}^{n+1} \\
+\left(\bar{a}_{p}+\frac{\Delta t}{h_{x}}+\frac{\Delta t}{2} \bar{\sigma}_{p}\right) v_{i-1, j, p+1}^{n+1}+\left(\bar{a}_{p}-\frac{\Delta t}{h_{x}}+\frac{\Delta t}{2} \bar{\sigma}_{p}\right) v_{i, j, p+1}^{n+1} .
\end{array}
$$

Finally, the termination conditions (41) yield explicit updates of the form

$$
\begin{array}{r}
\left(1+\frac{c \Delta t}{h_{x}}\right) \\
v_{i, j, P}^{n+1}=v_{i, j, k, Q}^{n}+v_{i-1, j, P}^{n}-v_{i-1, j, P}^{n+1} \\
+\frac{c \Delta t}{h_{x}}\left(v_{i-1, j, P}^{n+1}-v_{i, j, P}^{n}+v_{i-1, j, P}^{n}\right) .
\end{array}
$$

for the $\mathrm{x}$-direction.

\section{Appendix B. Explicit Procedure for the Yee Scheme}

We will consider the Yee Scheme as described in Sec. 3 with a maximum of $n_{x}, n_{y}$, and $n_{z}$ grid points in the $x, y$, and $z$ directions respectively. In 
particular, there are $\left(n_{x}-1, n_{y}, n_{z}\right)$ grid points for the $E_{x}$ component and $\left(n_{x}, n_{y}-1, n_{z}-1\right)$ grid points for the $H_{x}$, for example, due to the staggered grid.

\section{Appendix B.1. DAB Face Updates}

For faces with DAB boundary conditions that are not adjacent to other $\mathrm{DAB}$ faces, we only require the DAB updates for the tangential $\mathbf{E}$ field components. For example, we will consider the left boundary face in the $x$-direction. In this case, the tangential components are $E_{y}$ and $E_{z}$, so we introduce a three point layer of auxiliary variables that overlap the computational domain by two points for each of the components:

$$
\begin{gathered}
\left(\tilde{E}_{y}\right)_{i, j+1 / 2, k, p}^{1 / 2}=0, \quad i=0,1,2, \quad j=1, \ldots, n_{y}-1, \\
k=1, \ldots, n_{z}, \quad p=0, \ldots, P, \\
\left(\tilde{E}_{z}\right)_{i, j, k+1 / 2, p}^{1 / 2}=0, \quad i=0,1,2, \quad j=1, \ldots, n_{y}, \\
k=1, \ldots, n_{z}-1, \quad p=0, \ldots, P .
\end{gathered}
$$

We next apply the DAB updates as described for scalar waves in Sec. 2.3 . In particular, assuming we have evolved the interior values with the Yee scheme updates to time $t=(n+1 / 2) \Delta t$ for the $\mathbf{E}$ fields, we first copy the last plane of points with updates into the auxiliary variables:

$$
\begin{aligned}
& \left(\tilde{E}_{y}\right)_{2, j+1 / 2, k, 0}^{n+1 / 2}=\left(E_{y}\right)_{2, j+1 / 2, k}^{n+1 / 2}, \\
& \left(\tilde{E}_{z}\right)_{2, j, k+1 / 2,0}^{n+1 / 2}=\left(E_{z}\right)_{2, j, k+1 / 2}^{n+1 / 2} .
\end{aligned}
$$

Next, we update the interior of the DAB layer using the second order, centered difference approximation to the wave equation:

$$
\begin{array}{r}
\left(D_{t}^{+} D_{t}^{-}-c^{2}\left(D_{x}^{+} D_{x}^{-}+D_{y}^{+} D_{y}^{-}+D_{z}^{+} D_{z}^{-}\right)\right)\left(\tilde{E}_{y}\right)_{1, j+1 / 2, k, p}^{n-1 / 2}=0, \\
j=2, \ldots, n_{y}-2, \quad k=2, \ldots, n_{z}-1, \quad p=0, \ldots, P, \\
\left(D_{t}^{+} D_{t}^{-}-c^{2}\left(D_{x}^{+} D_{x}^{-}+D_{y}^{+} D_{y}^{-}+D_{z}^{+} D_{z}^{-}\right)\right)\left(\tilde{E}_{z}\right)_{1, j, k+1 / 2, p}^{n-1 / 2}=0, \\
j=2, \ldots, n_{y}-1, \quad k=2, \ldots, n_{z}-2, \quad p=0, \ldots, P .
\end{array}
$$

We note that this gives the 3D equivalent of A.1, which provides explicit updates for $\left(\tilde{E}_{y}\right)_{1, j+1 / 2, k, p}^{n+1 / 2}$ and $\left(\tilde{E}_{z}\right)_{1, j, k+1 / 2, p}^{n+1 / 2}$. We can also handle the adjacent boundary conditions here. For illustration, we consider the adjacent 
boundaries to be perfect electric conductors (PEC). PEC boundaries can be enforced in the Yee scheme by setting homogeneous Dirichlet conditions on the tangential $\mathbf{E}$ field components to the boundary and homogeneous Neumann conditions on the normal $\mathbf{E}$ field component. Since the DAB updates have not been used on the boundaries, the tangential components should already be correctly set to 0 . However, we need to deal with the normal components. We do this by substituting the discrete approximation to the homogeneous Neumann condition into the discrete wave equation updates to get

$$
\begin{aligned}
& D_{t}^{+} D_{t}^{-}\left(\tilde{E}_{y}\right)_{1, \frac{3}{2}, k, p}^{n-1 / 2}-c^{2}\left(\left[D_{x}^{+} D_{x}^{-}+D_{z}^{+} D_{z}^{-}\right]\left(\tilde{E}_{y}\right)_{1, \frac{3}{2}, k, p}^{n-1 / 2}\right. \\
& \left.+h_{y}^{-2}\left[-\left(\tilde{E}_{y}\right)_{1, \frac{3}{2}, k, p}^{n-1 / 2}+\left(\tilde{E}_{y}\right)_{1, \frac{5}{2}, k, p}^{n-1 / 2}\right]\right)=0, \\
& k=2, \ldots, n_{z}-1, \quad p=0, \ldots, P, \\
& D_{t}^{+} D_{t}^{-}\left(\tilde{E}_{y}\right)_{1, n_{y}-1 / 2, k, p}^{n-1 / 2}-c^{2}\left(\left[D_{x}^{+} D_{x}^{-}+D_{z}^{+} D_{z}^{-}\right]\left(\tilde{E}_{y}\right)_{1, n_{y}-1 / 2, k, p}^{n-1 / 2}\right. \\
& \left.+h_{y}^{-2}\left[-\left(\tilde{E}_{y}\right)_{1, n_{y}-1 / 2, k, p}^{n-1 / 2}+\left(\tilde{E}_{y}\right)_{1, n_{y}-\frac{3}{2}, k, p}^{n-1 / 2}\right]\right)=0 \\
& k=2, \ldots, n_{z}-1, \quad p=0, \ldots, P, \\
& D_{t}^{+} D_{t}^{-}\left(\tilde{E}_{z}\right)_{1, j, \frac{3}{2}, p}^{n-1 / 2}-c^{2}\left(\left[D_{x}^{+} D_{x}^{-}+D_{y}^{+} D_{y}^{-}\right]\left(\tilde{E}_{z}\right)_{1, j, \frac{3}{2}, p}^{n-1 / 2}\right. \\
& \left.+h_{z}^{-2}\left[-\left(\tilde{E}_{z}\right)_{1, j, \frac{3}{2}, p}^{n-1 / 2}+\left(\tilde{E}_{z}\right)_{1, j, \frac{5}{2}, p}^{n-1 / 2}\right]\right)=0, \\
& j=2, \ldots, n_{y}-1, \quad p=0, \ldots, P, \\
& D_{t}^{+} D_{t}^{-}\left(\tilde{E}_{z}\right)_{1, j, n_{z}-1 / 2, p}^{n-1 / 2}-c^{2}\left(\left[D_{x}^{+} D_{x}^{-}+D_{y}^{+} D_{y}^{-}\right]\left(\tilde{E}_{z}\right)_{1, j, n_{z}-1 / 2, p}^{n-1 / 2}\right. \\
& \left.+h_{z}^{-2}\left[-\left(\tilde{E}_{z}\right)_{1, j, n_{z}-1 / 2, p}^{n-1 / 2}+\left(\tilde{E}_{z}\right)_{1, j, n_{z}-\frac{3}{2}, p}^{n-1 / 2}\right]\right)=0 \\
& j=2, \ldots, n_{y}-1, \quad p=0, \ldots, P,
\end{aligned}
$$


Next, we can apply the Sommerfeld termination conditions:

$$
\begin{array}{r}
\left(D_{t}^{+} A_{x}^{+}-c D_{x}^{+} A_{t}^{+}\right)\left(\tilde{E}_{y}\right)_{0, j+1 / 2, k, P}^{n-1 / 2}=0 \\
j=1, \ldots, n_{y}-1, \quad k=1, \ldots, n_{z} \\
\left(D_{t}^{+} A_{x}^{+}-c D_{x}^{+} A_{t}^{+}\right)\left(\tilde{E}_{z}\right)_{0, j, k+1 / 2, P}^{n-1 / 2}=0 \\
j=1, \ldots, n_{y}, \quad k=1, \ldots, n_{z}-1 .
\end{array}
$$

Note that there is a sign change from the explicit update formula A.4 because the outward normal from the face we are considering is $x=-1$ whereas A.4 are given for the outward normal $x=1$.

Now we can apply the CRBC recursions (again, noting the sign change due to the outward normal direction):

$$
\begin{array}{r}
\left(\bar{a}_{j} D_{t}^{+} A_{x}^{+}+D_{x}^{+} A_{t}^{+}+\bar{\sigma}_{j} A_{t}^{+} A_{x}^{+}\right)\left(\tilde{E}_{y}\right)_{i, j+1 / 2, k, p+1}^{n-1 / 2}= \\
\left(a_{j} D_{t}^{+} A_{x}^{+}-D_{x}^{+} A_{t}^{+}+\sigma_{j} A_{t}^{+} A_{x}^{+}\right)\left(\tilde{E}_{y}\right)_{i, j+1 / 2, k, p}^{n-1 / 2}, \\
i=0,1, \quad j=1, \ldots, n_{y}-1, \quad k=1, \ldots, n_{z}, \\
\left(\bar{a}_{j} D_{t}^{+} A_{x}^{+}+D_{x}^{+} A_{t}^{+}+\bar{\sigma}_{j} A_{t}^{+} A_{x}^{+}\right)\left(\tilde{E}_{z}\right)_{i, j, k+1 / 2, p+1}^{n-1 / 2}= \\
\left(a_{j} D_{t}^{+} A_{x}^{+}-D_{x}^{+} A_{t}^{+}+\sigma_{j} A_{t}^{+} A_{x}^{+}\right)\left(\tilde{E}_{z}\right)_{i, j, k+1 / 2, p}^{n-1 / 2}, \\
i=0,1, \quad j=1, \ldots, n_{y}, \quad k=1, \ldots, n_{z}-1 .
\end{array}
$$

Finally, we can provide the updated values to the interior Yee scheme updater:

$$
\begin{aligned}
& \left(E_{y}\right)_{1, j+1 / 2, k}^{n+1 / 2}=\left(\tilde{E}_{y}\right)_{1, j+1 / 2, k, 0}^{n+1 / 2} \\
& \left(E_{z}\right)_{1, j, k+1 / 2}^{n+1 / 2}=\left(\tilde{E}_{z}\right)_{1, j, k+1 / 2,0}^{n+1 / 2}
\end{aligned}
$$

\section{Appendix B.2. DAB Edges}

If there are two or more adjacent DAB layers, we cannot update points at the intersecting edge as described. In particular, we cannot apply updates analogous to (B.7) B.10). Additionally, to update an edge we require auxiliary data from the normal components on the intersecting faces. To get this data, we simply perform DAB face updates described in Appendix B.1 
on the normal component; however, we omit the last step of copying the values back into the interior because the Yee scheme should have correctly updated the points.

To illustrate the edge conditions, we'll consider the intersection of two faces: one with a outward normal $x=-1$ and the other with the outward normal $y=1$. We will assume that we have updated all of the auxiliary variables that we can on each of the faces, so we have

$$
\begin{gathered}
\left(\tilde{E}_{x}\right)_{i+1 / 2, j, k, p}^{n+1 / 2}, \quad i=0,1,2 \quad j=1, \ldots, n_{y}-1, \quad k=1, \ldots, n_{z}, \\
\left(\tilde{E}_{y}\right)_{j, j+1 / 2, k, p}^{n+1 / 2}, \quad i=0,1,2 \quad j=1, \ldots, n_{y}-2, \quad k=1, \ldots, n_{z}, \\
\left(\tilde{E}_{z}\right)_{k, j, k+1 / 2, p}^{n+1 / 2}, \quad i=0,1,2 \quad j=1, \ldots, n_{y}-1, \\
k=1, \ldots, n_{z}-1, \\
\left(\hat{E}_{x}\right)_{i+1 / 2, j, k, q}^{n+1 / 2}, \quad i=2, \ldots, n_{x}-1 \quad j=n_{y}-1, n_{y}, n_{y}+1, \\
\left(\hat{E}_{y}\right)_{j, j+1 / 2, k, q}^{n+1 / 2}, \quad i=2, \ldots, n_{x} \quad j=n_{y}-2, n_{y}-1, n_{y}, \\
k=1, \ldots, n_{z}, \\
\left(\hat{E}_{z}\right)_{k, j, k+1 / 2, q}^{n+1 / 2}, \quad i=2, \ldots, n_{x} \quad j=n_{y}-1, n_{y}, n_{y}+1, \\
k=1, \ldots, n_{z}-1,
\end{gathered}
$$

where we use $\tilde{E}$ to denote the auxiliary variables on the face with normal $x=-1$ and $\hat{E}$ to denote the auxiliary variables on the face with normal $y=1$. For the $E_{x}$ components, we need to calculate updates at the spatial location $\left(1, n_{y}, k\right)$ for $k=1, \ldots, n_{z}$. Similarly, we need updates at $\left(1, n_{y}-1, k\right)$ and $\left(1, n_{y}, k\right)$ for $E_{y}$ and $E_{z}$, respectively. In each case, the process is the same, so we only consider the case for the $E_{z}$ component. We begin by introducing a doubly indexed set of auxiliary variables that overlaps the auxiliary variables $\tilde{E}_{z}$ and $\hat{E}_{z}$

$$
\begin{aligned}
\left(\bar{E}_{z}\right)_{i, j, k+1 / 2, p, q}^{1 / 2} & =0, \quad i=\{0,1,2\}, \quad j=n_{y}-1, n_{y}, n_{y}+1 \\
k & =1, \ldots, n_{z}-1, \quad p=0, \ldots, P, \quad q=0, \ldots, P,
\end{aligned}
$$

where we assume that there are the same number $(P+1)$ of auxiliary variables on each of the faces. We will additionally assume that the auxiliary 
equations on each of the faces uses the same CRBC parameters although in general this need not be the case. We use the convention that the $p$ index corresponds to recursions in the $x$ direction and the $q$ index corresponds to recursions in the $y$ direction.

We input the data from the faces into the edge auxiliary variables:

$$
\begin{aligned}
\left(\bar{E}_{z}\right)_{1, n_{y}-1, k+1 / 2, p, 0}^{n+1 / 2} & =\left(\tilde{E}_{z}\right)_{1, n_{y}-1, k+1 / 2, p}^{n+1 / 2} \\
\left(\bar{E}_{z}\right)_{2, n_{y}, k+1 / 2,0, q}^{n+1 / 2} & =\left(\hat{E}_{z}\right)_{2, n_{y}, k+1 / 2, q}^{n+1 / 2}
\end{aligned}
$$

Next we update the interior points for all of the auxiliary variables using the wave equation

$$
\begin{array}{r}
D_{t}^{+} D_{t}^{-}\left(\bar{E}_{z}\right)_{1, n_{y}, k+1 / 2, p, q}^{n-1 / 2} \\
-c^{2}\left(D_{x}^{+} D_{x}^{-}+D_{y}^{+} D_{y}^{-}+D_{z}^{+} D_{z}^{-}\right)\left(\bar{E}_{z}\right)_{1, n_{y}, k+1 / 2, p, q}^{n-1 / 2}=0 \\
k=2, \ldots, n_{z}-2, \quad p=0, \ldots, P, \quad q=0, \ldots, P .
\end{array}
$$

We additionally apply any adjacent boundary conditions here as described in Appendix B.1 for the face updates. Then, we can apply the Sommerfeld termination conditions:

$$
\begin{array}{r}
\left(D_{t}^{+} A_{x}^{+}-c D_{x}^{+} A_{t}^{+}\right)\left(\bar{E}_{z}\right)_{0, n_{y}, k+1 / 2, P, q}^{n-1 / 2}=0 \\
k=1, \ldots, n_{z}-1, \quad q=0, \ldots, P . \\
\left(D_{t}^{+} A_{y}^{+}+c D_{y}^{+} A_{t}^{+}\right)\left(\bar{E}_{z}\right)_{1, n_{y}, k+1 / 2, p, P}^{n-1 / 2}=0 \\
k=1, \ldots, n_{z}-1, \quad p=0, \ldots, P .
\end{array}
$$

Now we can apply the CRBC recursions (again, noting the sign change due to the outward normal direction):

$$
\begin{array}{r}
\left(\bar{a}_{j} D_{t}^{+} A_{x}^{+}+D_{x}^{+} A_{t}^{+}+\bar{\sigma}_{j} A_{t}^{+} A_{x}^{+}\right)\left(\bar{E}_{z}\right)_{i, n_{y}, k+1 / 2, p+1, q}^{n-1 / 2}= \\
\left(a_{j} D_{t}^{+} A_{x}^{+}-D_{x}^{+} A_{t}^{+}+\sigma_{j} A_{t}^{+} A_{x}^{+}\right)\left(\bar{E}_{z}\right)_{i, n_{y}, k+1 / 2, p, q}^{n-1 / 2}, \\
i=0,1, \quad k=1, \ldots, n_{z}-1 . \\
\left(\bar{a}_{j} D_{t}^{+} A_{j}^{+}-D_{j}^{+} A_{t}^{+}+\bar{\sigma}_{j} A_{t}^{+} A_{j}^{+}\right)\left(\bar{E}_{z}\right)_{1, j, k+1 / 2, p, q+1}^{n-1 / 2}= \\
\left(a_{j} D_{t}^{+} A_{y}^{+}+D_{y}^{+} A_{t}^{+}+\sigma_{j} A_{t}^{+} A_{y}^{+}\right)\left(\bar{E}_{z}\right)_{1, n_{y}, k+1 / 2, p, q}^{n-1 / 2}, \\
j=n_{y}-1, n_{y}, \quad k=1, \ldots, n_{z}-1 .
\end{array}
$$


Finally, we can provide the updated values to the interior Yee scheme updater and the auxiliary variables on the faces:

$$
\begin{gathered}
\left(E_{z}\right)_{1, n_{y}, k+1 / 2}^{n+1 / 2}=\left(\bar{E}_{z}\right)_{1, n_{y}, k+1 / 2,0,0}^{n+1 / 2}, \\
\left(\tilde{E}_{z}\right)_{1, n_{y}, k+1 / 2, p}^{n+1 / 2}=\left(\bar{E}_{z}\right)_{1, n_{y}, k+1 / 2, p, 0}^{n+1 / 2} \\
\left(\hat{E}_{z}\right)_{1, n_{y}, k+1 / 2, q}^{n+1 / 2}=\left(\bar{E}_{z}\right)_{1, n_{y}, k+1 / 2,0, q}^{n+1 / 2}
\end{gathered}
$$

We note that corners are handled analogously by introducing a triply index set of auxiliary variables. 